\title{
Modelling of Mean Flame Shape During Premixed Flame Flashback in Turbulent Boundary Layers
}

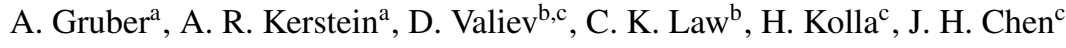 \\ ${ }^{a}$ SINTEF Energy Research, Trondheim, Norway. \\ ${ }^{b}$ Princeton University, Princeton, New Jersey \\ ${ }^{c}$ Combustion Research Facility, Livermore, California.
}

\begin{abstract}
Direct numerical simulations of freely-propagating premixed flames in the turbulent boundary layer of fully-developed turbulent channel flows are used for a priori validation of a new model that aims to describe the mean shape of the turbulent flame brush during flashback. Comparison with the DNS datasets, for both fuel-lean and fuel-rich mixture conditions and for Damköhler numbers lower and larger than unity, shows that the model is able to capture the main features of the flame shape. Although further a priori and a posteriori validation is required, particularly at higher Reynolds numbers, this new simple model seems promising and can potentially have impact on the design process of industrial combustion equipment.
\end{abstract}

Keywords:

Turbulent Channel Flow, Flame Shape Model, Turbulent Premixed Flame, Flame Propagation 


\section{Introduction}

Efforts aimed at the reduction in global emissions of greenhouse-gases and at the control of local emissions of pollutants are increasingly important for the energy sector. A considerable reduction in fuel consumption, due to increased efficiency, and in pollutant emissions, because of low flame temperature and without increase in the energy penalty intrinsic to dilution, can be achieved in stationary gas turbines utilizing leanpremixed (LPM) combustion technology [1]. Among the many challenges in enabling LPM combustion technology for gas turbine applications, accurate prediction of flame flashback, especially for non-conventional and highly reactive hydrogen-rich fuels [2], stands out as a key requirement for complete understanding of the complex physical processes that are of fundamental importance in burner design and optimization. Recent studies, involving high-resolution experimental measurements and direct numerical simulations (DNS) [3, 4], focused on the characterization of flashback for premixed, preheated hydrogen-air flames in turbulent boundary layers, and have shown that one of the principal assumptions behind the widely-used flashback model of Lewis and Von Elbe [5] is flawed. Indeed, this pioneering model, dating back to 1943 , in determining the critical velocity gradient for the onset of flashback erroneously assumes that the premixed flame, during its flashback in the wall boundary layer, has no effect on the approaching flow of the reactants while the recent investigations mentioned above have revealed the presence of flow reversals, induced by the flame in the viscous layer $\left(y^{+} \lesssim 20\right)$, located immediately upstream of the flame surface regions that are convex towards the reactants [4]. The implications of this finding range from a radically different picture about the mechanism of boundary layer flashback to the renewed need for a near-wall flame propagation model that correctly takes this new information into account. Other early studies, building on the methodology proposed in [5], have tried to chart the flashback behavior of premixed flames in the transition from laminar to the more interesting case of turbulent flows [6,7] and have reported empirical observation of considerable increases in the critical velocity gradients in the presence of turbulence; however, they have failed to clearly explain the reason for such increase in the context of a model that is ultimately based on a nearwall velocity balance within the quasi-laminar viscous layer. More recent studies [8, 9] have taken into account local Lewis number and flame curvature effects on the onset of laminar boundary layer flashback but have not considered near-wall flame surface perturba- tion and curvature in the plane parallel to the wall that results from the interaction of the flame with the boundary layer streaks and that is instrumental to the creation of the flow reversals. Similarly to the classical work [5], the vast majority of theoretical models on turbulent combustion employs the limit of zero gas expansion, however a number of recent theoretical works take realistic gas expansion into account $[10,11]$. Following the latter approach in the attempt to fill existing gaps, we propose here a new model for the turbulent flame shape for premixed combustion in wall boundary layers that takes into account gas expansion and we provide $a$ priori validation of the model based on the analysis of relevant DNS data. The remainder of this paper is organized as follows: Section 2 presents the new flashback model. Section 3 describes the DNS computations. Section 4 illustrates the a priori validation of the model. Finally, Section 5 summarizes the main findings and provides an outline of future planned work.

\section{Flame Shape Model Description}

A model of the mean shape of a turbulent premixed flame in turbulent channel flow is formulated. In general, the mean location of the turbulent flame brush is represented as an interface that propagates, relative to the mean flow, following Huygens propagation picture and at the mean turbulent burning velocity $S_{T}$, whose parameterization is described in Section 4. In that parameterization, $S_{T}$ depends on the laminar flame speed $S_{L}$, on the density ratio $D=\rho_{u} / \rho_{b}$, where the subscripts $u$ and $b$ denote unburnt and burnt conditions respectively, and on the rms velocity fluctuation $u^{\prime}$. For the present anisotropic case, $u^{\prime}$ is interpreted as the square root of twice the mean turbulent kinetic energy per unit mass. $u^{\prime}$ is taken to be a known function of the wallnormal coordinate $y$ based on a turbulent channel flow DNS database [12]. Steady state mean flame propagation is assumed. This implies a balance of the mean flow and the propagation of the interface toward the flow. The mean flow $U_{m}(y)$, which is streamwise and $y$ dependent, is likewise obtained from the DNS database [12]. A sketch of the notional flame-in-channel configuration is given in Fig. 1. By symmetry, the interface normal at the center-plane $y=h$ (assuming walls at 0 and $2 h$ ) is streamwise oriented, so the mean interface velocity at $y=h$ is $V_{f}=U_{m}(h)-S_{T}(h)$. This mean lab-frame stabilization speed, $V_{f}$, can also be expressed locally as $V_{f}(y)=\left(S_{d}(y)+U_{n}(y)\right)_{x}$ where $S_{d}(y)$ and $U_{n}(y)$ are the local flame surface displacement speed relative to the fluid and the local (flame surface normal) fluid velocity respectively [13]. Here, the flame- 


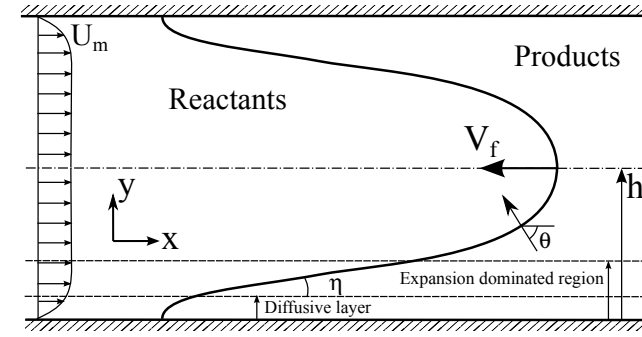

Figure 1: A sketch of the notional flame in the channel.

normal direction is assumed to be a continuous function of $y$, which is not necessarily valid; see below. Steady state mean flame shape implies that the streamwise interface displacement rate $V_{f}$ is, in the mean, independent of $y$. For $y \neq h$, the flame normal is not streamwise oriented. A relationship valid for all $y$ is $V_{f}(y)=U_{m}(y)-S_{T x}(y)$, where $S_{T x}(y)$ is the upstream (opposing the mean flow) displacement rate of the interface resulting from propagation at speed $S_{T}(y)$ in the flame-normal direction. Denote the angle of the interface normal relative to the upstream direction as $\theta$. Consider a locally planar interface for given $\theta$. For $\theta \neq \pi / 2$, displacement of this interface by a given distance $D_{n}$ in the interface-normal direction results in interface upstream displacement $D_{n} / \cos \theta$ at given $y$. This corresponds to $S_{T x}(y)=S_{T}(y) / \cos \theta$. Note that this gives the required behaviors $S_{T x}(y)=S_{T}(y)$ for $\theta=0$ and divergence of $S_{T x}(y)$ for $\theta \rightarrow \pi / 2$. Combining results,

$$
U_{m}(h)-S_{T}(h)=U_{m}(y)-S_{T}(y) / \cos \theta(y)
$$

is obtained. Other self-consistent solutions can be obtained by allowing a discontinuity of the interfacenormal orientation (i.e., a cusp) at the center plane. Then a family of self-consistent solutions $U_{m}(h)-$ $S_{T}(h) / \cos |\theta(h)|=U_{m}(y)-S_{T}(y) / \cos \theta(y)$, parameterized by $|\theta(h)|$, is obtained. Solutions with nonzero $|\theta(h)|$ can be valid for laminar flames that often show a centerline cusp [14] but, as the present DNS dataset seems to indicate, they can also apply to turbulent flames. The presence of a cusp at the channel centerline in the turbulent case is consistent with wall-controlled flashback, where the leading-edge of the flame front advances rapidly upstream near the wall while downstream of the leading edge, the flame merely follows, closing the gap as the symmetric flames converge at the centerline. Equation 1 uniquely determines $|\theta(y)|$. Requiring $\theta$ to be a continuous function of $y$ (i.e., ruling out cusps) determines the function $\theta(y)$ up to an overall sign. This sign ambiguity allows the freedom to apply a stream- wise flip to the flame shape, i.e., the transformation $x \rightarrow-x$. This ambiguity is removed empirically based on the observation that walls are leading rather than lagging points of the flame for cases of interest. At the wall, e.g. $y=0, U_{m}(y)$ approaches zero and $S_{T}$ approaches $S_{L}$, so $\cos \theta(y)$ has the $y$-independent value $-S_{L} / V_{f}$ in some vicinity of $y=0$. To incorporate thermal expansion into the analysis, consequences of the inequality $\rho_{b} \ll \rho_{u}$ are noted. Dilatation at the interface is the result of a local pressure buildup that exerts forces in all directions. Owing to the much lower density of the products relative to the reactants, the pressure buildup accelerates the reactants much less than the products, so it is reasonable to neglect dilatation effects on the reactants as a rough approximation. Close to the wall, however, there is a countervailing effect. In general, $U_{m}(h)$ and $S_{T}(h)$ are larger than $S_{L}$, and as noted, $U_{m}(h)$ is substantially greater than $S_{T}(h)$, so $V_{f}$ is larger than $S_{L}$. Then $|\theta|$ in the near-wall region approaches $\pi / 2$, implying that the product layer near the wall forms a thin wedge. In this configuration, dilatation in the product layer requires much larger fluid displacements if confined to the product layer than if the dilatation can also displace the overlying reactant in the wall-normal direction. Therefore, not considering for now the flame leading edge (where diffusive effects require modification of the Huygens propagation picture; see below), it is expected that a significant if not predominant portion of the dilatation occurs in the wall-normal direction. For estimation purposes it is assumed that all the near-wall dilatation is wall-normal. A further approximation is that this dilatation is the dominant mechanism that tilts the flame normal away from the streamwise direction near the wall. Then treating dilatation as the only such mechanism, it follows that $\tan |\theta(0)|=V_{f} /\left(D S_{L}\right)$. Then the contact angle $\eta=\pi / 2-|\theta(0)|$ of the interface with the wall obeys $\tan \eta=D S_{L} / V_{f}$. Comparing this to the estimate $\tan \eta=S_{L} / V_{f}$ for the constant-density case, the inequality $D \gg 1$ confirms that dilatation is the main cause of interface tilt near the wall. At the leading edge of the flame, when certain conditions are met locally by the approaching flow [4], dilatation of the burning fluid near the wall displaces the nearby reactants not only upward/downward (in the wall-normal direction) but also upstream, originating characteristics flow reversals. This, in turn, results in a blockage effect by the flame on the approaching flow of the reactants that are deflected towards the channel centerline, finally producing an acceleration of the bulk flow far from the walls. Taking into account the effect of the flow reversals induced by the flame, the subsequent near-wall blockage and the acceleration of the reactants flow near the cen- 
terline implies that a (rough) estimate of the blockage thickness has to be provided in the model. In order to achieve this, the Huygens propagation picture is modified by considering diffusive effects very close to the the wall. The wall-normal diffusive spreading of any scalar controlling flame advancement obeys the scaling $y \sim(\kappa t)^{1 / 2}$, where $\kappa$ is the molecular diffusivity of the scalar. The resulting flame shape $y(x)$ is obtained by relating $t$ to $x$ using the relation $t=x / S_{L}$ based on the laminar flame speed $S_{L}$ (reasonable assumption in the viscous layer, where $\left.u^{\prime} \sim 0\right)$. Then $y \sim\left(\kappa x / S_{L}\right)^{1 / 2}$, giving $d y / d x \sim x^{-1 / 2}$, where $d y / d x$ corresponds to $\tan \eta$. This shows that $\eta=\pi / 2$ at the leading point of the flame, which is at $x=0$ in this notation, with gradually decreasing $\eta$ thereafter (at larger $x$ ). A slope-matching condition is applied. The diffusive layer extends from the wall to the $y$ location at which $d y / d x$ given by the diffusive scaling equals $D S_{L} / V_{f}$. This matching condition provides an estimate for the blockage thickness as $y_{b} \sim\left(\kappa / S_{L}\right)^{1 / 2} \cdot D S_{L} / V_{f}$ that is utilized in the model. The result that the flame is streamwise oriented at the wall is based on a notional flame-controlling scalar that emanates from the wall. Depending on the wall boundary condition, other flame orientations are possible at the wall. For example, an isothermal wall that is cooler than the flame can be a sink rather than a source of the relevant scalar, with the possible result that the leading point of the flame is slightly offset from the wall, as indicated by numerical results. Thermal expansion within the diffusion layer is likely to be significantly if not primarily in the streamwise direction, suggesting additional complexity of the structure of this layer. The effect of the near-wall blockage on the core flow (the region that is farther than $y_{b}$ from either wall) is represented in the shape model by uniformly compressing the spatial coordinate of the DNS-derived wall-normal profiles $u^{\prime}$ and $U_{m}$ to match the span $\left[y_{b}, 2 h-y_{b}\right]$ of the core flow. This is the only effect of the core-flow submodel on the flame shape model. Mean flow streamlines (not shown) from the flame simulations described in Sec. 3 suggest uniform flow compression induced by wall blockage. This is one of two model representations of thermal expansion effects. The other is the $D$ dependence of $S_{T}$.

\section{DNS of Premixed Flame Propagation in Turbu- lent Channel Flow}

The Navier-Stokes equations for a compressible reactive, fluid are solved in a three-dimensional computational domain to simulate the upstream propagation of two non-anchored premixed $\mathrm{H}_{2}$-air flames in fully developed turbulent channel flow at fuel-air equivalence ratios of $\phi=0.55$ and $\phi=1.5$, denoted here as case TCF055 and case TCF150 respectively. The latter case, at fuel-rich mixture conditions and Damköhler number larger than unity ( $D a \sim 1.22)$, is described in more detail in a previous paper [4]. The former case, at fuel-lean mixture conditions and Damköhler number smaller than unity ( $D a \sim 0.7)$, is more relevant to gas turbine application (lean stoichiometry, low Damköhler number) and it is presented here for the first time. Two auxiliary inert direct simulations of a turbulent Poiseuille flow at $R e_{\tau} \sim 180$ are used for temporal sampling, at a fixed streamwise position, of the velocity components that are fed at the inlet plane of the reacting DNS. The three spatial directions in this configuration are the streamwise direction $(\mathrm{X})$, wall normal direction $(\mathrm{Y})$ and spanwise direction (Z). The temperature of the wall and of the reactants is set to $750[K]$ for both reactive cases TCF055 and TCF150 and the pressure in the entire domain is set to $2 \mathrm{~atm}$. The chemical reactions in the gas phase are described by a detailed mechanism for hydrogen combustion in air [15]. Navier-Stokes Characteristic Boundary Conditions (NSCBC) are implemented based on the original formulation of [16] and on the later improvements described in [17] and [18]. The boundary conditions are non-reflecting at the inflow $(x=0)$ and outflow $\left(x=L_{x}\right)$ planes, no-slip isothermal solid surface at the wall boundaries $\left(y=0\right.$ and $\left.y=L_{y}\right)$, and periodic in the spanwise direction $\left(z=0\right.$ and $\left.z=L_{z}\right)$. The present NSCBC implementation allows only small variations around the nominal values of the time-varying velocity components imposed at the inlet plane and will therefore affect the upstream flame propagation in its immediate vicinity [4]. The wall is assumed to be impermeable, so the wall-normal mass flux of all chemical species is set to zero. The Reynolds number of the approach flows is $R e_{0} \sim 3200$ for both TCF055 and TCF150, based on the channel centerline velocities $U_{c}^{1}$ and $U_{c}^{2}$ of the fresh reactants and the channel halfwidths, $h_{1}$ and $h_{2}$, where subscripts 1 and 2 denote case TCF055 and TCF150 respectively. This approximately corresponds to a friction Reynolds number $R e_{\tau} \sim 180$, as in the non-reacting auxiliary simulations. All turbulent quantities used below for non-dimensionalization characterize the turbulent flow of the fresh reactants upstream of the flame. The Damköhler numbers (ratio of a chemical to dynamical time scale) that characterize the turbulent flames are $D a_{1} \sim 0.69$ and $D a_{2} \sim 1.22$. As suggested in [19], these Damköhler numbers are based on the freely propagating one-dimensional laminar flame time scale $\left(t_{l 1}=\delta_{l 1} / S_{l 1} \sim 4.8 e^{-05}[s]\right.$ and 
Table 1: DNS parameters for the reactive cases: $h$ is the channel half-width, $\delta_{v}$ the viscous length scale and $\Delta^{+}$is the non-dimensional grid resolution (normalized by $\delta_{v}$ ).

\begin{tabular}{|l|c|c|c|c|c|c|c|c|c|}
\hline Case & $\phi$ & $U_{c}$ & $h$ & $L_{x} \times L_{y} \times L_{z}$ & $N_{x} \times N_{y} \times N_{z}$ & $\delta_{v}$ & $\Delta^{+}$ & $S_{l} / U_{c}$ & $D a$ \\
\hline TCF055 & 0.55 & $20(\mathrm{~m} / \mathrm{s})$ & $6(\mathrm{~mm})$ & $10 h \times 2 h \times 6 h$ & $2400 \times 480 \times 1440$ & $3.4 e^{-05}(\mathrm{~m})$ & 0.73 & 0.35 & 0.69 \\
$\mathrm{TCF} 150$ & 1.50 & $25(\mathrm{~m} / \mathrm{s})$ & $5(\mathrm{~mm})$ & $5 h \times 2 h \times 3 h$ & $1000 \times 400 \times 600$ & $2.8 e^{-05}(\mathrm{~m})$ & 0.89 & 0.55 & 1.22 \\
\hline
\end{tabular}

$\left.t_{l 2}=\delta_{l 2} / S_{l 2} \sim 1.9 e^{-05}[s]\right)$ and on the wall time scale that is uniquely defined from the turbulent channel flow of the fresh reactants $\left(t_{w 1}=v_{1} / u_{\tau}^{2} \sim 3.3 e^{-05}[s]\right.$ and $\left.t_{w 2}=v_{2} / u_{\tau}^{2} \sim 2.3 e^{-05}[s]\right)$. In these expressions $u_{\tau}$ is the friction velocity, $v_{1}$ and $v_{2}$ are the kinematic viscosities of the fresh reactants, and $\delta_{l 1,2}$ and $S_{l 1,2}$ are the laminar flame thicknesses and laminar flame velocities at the two equivalence ratios. It should be noted that the flame thicknesses $\delta_{l 1,2}$ at $\phi=0.55$ and $\phi=1.5$ respectively are estimated in terms of the fuel reaction rate thickness. Other relevant parameters of the DNS are given in Tab. 1. The turbulent hydrogen-air reactant mixtures enters the channel from a non-reflecting inlet on the left boundary of the domain and approaches the flame in the streamwise direction while the burnt products leave the computational domain from a non-reflecting outlet on the right domain boundary, see Fig. 2. The reactive cases TCF055 and TCF150 are initialized by imposing a constant pressure equal to $2 \mathrm{~atm}$ throughout the domain and the instantaneous fluctuating velocity, density and temperature fields computed in the inert simulation. This procedure ensures that the flame encounters realistic approaching turbulence from the beginning of the simulation, thereby enabling a relatively short settling time. A planar premixed laminar flame is superimposed on the initial velocity field obtained from the auxiliary non-reacting simulation. Burnt adiabatic product conditions are imposed downstream of the flame. The initial streamwise velocity field component in the reacting simulations is adjusted, only once and prior to the start of the simulation, for compatibility with the lower density on the product side of the flame. A progress variable function $C$ is used in the initialization to map all points in the three-dimensional domain to a one-dimensional CHEMKIN PREMIX solution of the freely propagating $\mathrm{H}_{2}$-air flame configuration. The progress variable $C$ is a scalar parametrization of the reactive flow field, based on the hydrogen mass fraction, that is equal to zero in the fresh reactants and unity in the burnt products. The initialization technique described above yields a marginally incorrect initial pressure field. Therefore, an initial 'settling' time interval of approximately five times the channel acoustic transit time is allowed for the initial pres- sure fluctuations to exit the domain from the inlet and outlet boundaries. After an initial settling time interval $\Delta t^{s}$ approximately equal to $4.1 e^{-04}[s]$ for TCF055 and $1.9 e^{-04}[s]$ for TCF150, the turbulence-flame interaction is no longer affected by the initial pressure fluctuations and at this point the flames have been wrinkled by the approaching turbulence and have begun to propagate upstream. Please note that, according to the model from Lewis and Von Elbe [5], flashback should not take place under the present conditions as the nominal value of the critical velocity gradient is exceeded. Due to the

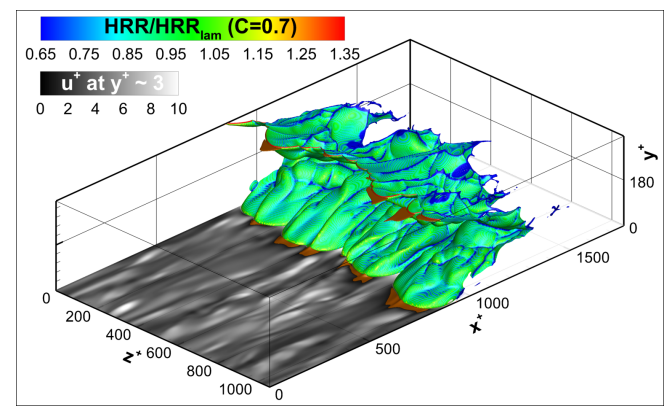

Figure 2: Instantaneous 3-D plot of the turbulent flame sheet during flashback visualized by the isosurface of of the progress variable $\mathrm{C}=0.7$ for case $\mathrm{TCF} 055$. Note the flow reversal pockets visualized by the gold-colored isosurfaces.

intrinsic transient characteristics of this particular flame configuration, results are sampled every 0.5 wall time units, $t_{w}$, to ensure sufficient sample sizes for statistical post-processing. The sampling interval is equivalent to approximately one channel convective transit time providing approximately 70 and 50 samples after the initial transient respectively for case TCF055 and TCF150. The samples relative to the time immediately before the flame reaches the region near the upstream inlet boundary of the domain are excluded from the averaging due to the effect of the boundary conditions on the flame shape and velocity. The numerical integration time step is fixed at a value, $\Delta t=4.0 e^{-09}[s]$ in the reactive cases corresponding to approximately 10000 and 7000 time steps per wall time unit, $t_{w 1}$ and $t_{w 2}$, respectively. The three-dimensional Cartesian grids utilized are uniform in all directions. The first point from the wall is at 
$0.7<y^{+}<0.9$ where the superscript + indicates nondimensionalization by the viscous length scale. There are 11 points within $y^{+}=10$ to satisfy the resolution requirements in the viscous layer [12]. The grid resolution is $\Delta x^{+}=\Delta y^{+}=\Delta z^{+} \sim 0.7$ for TCF055 and $\sim 0.9$ for TCF150. The grid is not stretched, not even in the wallnormal direction, in order to accurately represent the flame which requires high spatial resolution throughout the channel, including near the centerline. The parallel DNS code, S3D [20], is used to perform the present DNS. In addition to the previous flame-wall interaction studies [19, 4], S3D has been used for a range of studies, including premixed and non-premixed flames. S3D is written in FORTRAN 90 and uses the Message Passing Interface (MPI) for interprocess communication in parallel execution. Spatial derivatives are computed with an eighth-order, explicit, centered, finite-difference scheme (third-order one-sided stencils are used at the domain boundaries in the non-homogeneous directions) in conjunction with a tenth-order, explicit, spatial filter, as described in [21], to remove high frequency noise and reduce aliasing error. A fourth-order, six-stage, explicit Runge-Kutta scheme, described in [22], is used for time integration.

\section{A Priori Model Validation}

The DNS provides extremely detailed fundamental knowledge about the physical mechanism controlling boundary layer flashback of turbulent premixed flames. The present wall flashback model uses $U_{m}(y)$ and $u^{\prime}(y)$ from the Moser database [12] as input describing the approaching turbulent flow of the reactants. The two DNS databases TCF055 and TCF150 are processed to extract information about flame shape and velocity that is used to construct and validate the model a priori. The scat-

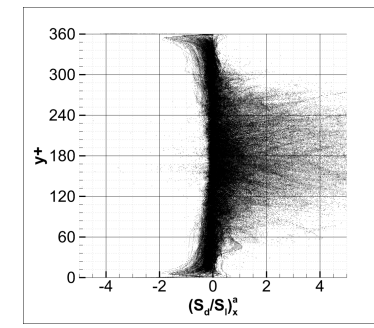

(a)

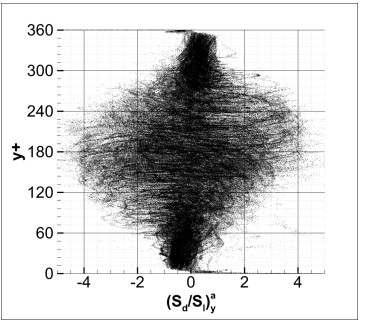

(b)
Figure 3: Scatter plots of the streamwise 3(a) and wall-normal 3(b) component of the lab-frame flame surface displacement speed $\left(S_{d} / S_{L}\right)^{a}$.

ter plot in Fig. 3(a) shows the complete TCF055 dataset for the streamwise component of the lab-frame flame surface velocity normalized by the laminar flame speed $\left(S_{d}+U_{n}\right)_{x} / S_{L}=\left(S_{d} / S_{L}\right)_{x}^{a}$ and clearly illustrates that the flame tongues in the near-wall region lead the flashback event with the largest streamwise velocity component values $\sim 2$. Near the channel centerline, the wallnormal component (in Fig. 3(b)) and z-component (not shown) attain large instantaneous values due to the large velocities of the underlying turbulent flow field but they are negligible in the mean. Interestingly, the former one peaks at $\sim 1(\sim-1)$ in the region of the flow closer to the upper (lower) wall, indicating a lack of turbulent flame acceleration in the wall-normal direction beyond the laminar flame velocity. A generalized flame shape

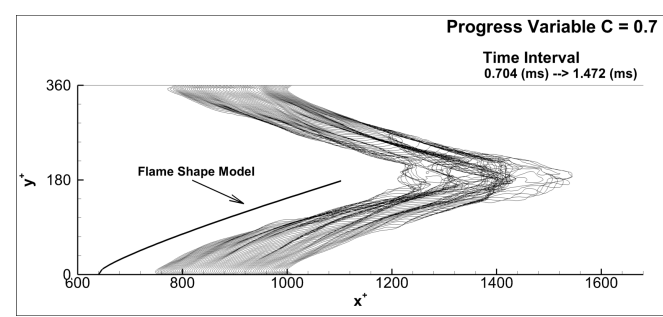

(a)

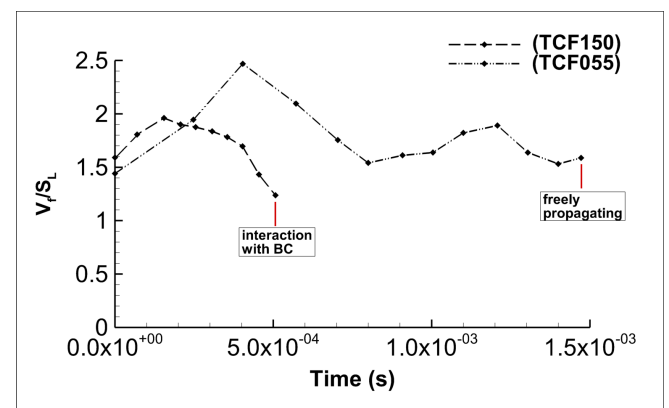

(b)

Figure 4: Time evolution and location of the spanwise-averaged flame surface shape compared with the modelled flame shape for case TCF055 4(a). Normalized front speed for case TCF055 and TCF150 4(b).

in the $X-Y$ plane, during its transient propagation in the turbulent channel flow, is obtained by averaging the reaction progress variable $C$ in the homogeneous spanwise direction and it is shown in Fig. 4(a) by the contours of $C=0.7$ for case TCF055 (after the initial transient). In the same plot, the modelled flame shape is also shown for comparison (thick line) and placed at an arbitrary position upstream of the DNS data. The local normals and, finally, the angles $\theta$ and $\eta=\pi / 2-\theta$ can be easily extracted from these spanwise-averaged curves and presented in Fig. 5 below. Observation of Fig. 4(a) pro- 
vides a first order estimate of the streamwise distance required by the two flame branches to reach the channel centerline and merge (streamwise span of the V-shaped flame), this distance being approximately 500 wall units or $17[\mathrm{~mm}]$ at all times. For a nominal laminar flame speed $S_{L}=0.35 \cdot U_{c}$ it takes a time $t_{l h}=h /\left(0.35 \cdot U_{c}\right)$ for an undisturbed laminar flame to reach the channel centerline, over that time period the flame front will have been transported downstream by the bulk flow a distance $U_{c} \cdot t_{l h}=h / 0.35$ or approximately $17[\mathrm{~mm}]$ for $h=6[\mathrm{~mm}]$. This confirms that $\left(S_{d} / S_{L}\right)_{y}^{a} \sim 1$. Also, the overall flashback front speed can be obtained by integration of the distance covered by the most upstream flame tongue at each instant for the given time interval, see Fig. 4(b) and the more detailed explanation provided in [4]. The flame front propagates upstream at a velocity $1.5 S_{L}<V_{f}<2.0 S_{L}$, confirming the indications from the scatter plot. Based on the consideration above, we define the lab-frame flashback velocity to be used in the model as $V_{f} \sim-A \cdot S_{L}$ where the value of the parameter $A$ (that should ideally be between 1.5 and 2$)$ is conveniently set using the expression $A=1+\left(D \cdot(D-1)^{2}\right) /\left(D^{3}+D^{2}+3 \cdot D-1\right)$ provided in [23]. Furthermore, we assume that the turbulent burning velocity has the following functional form $S_{T}=\left(\left(B \cdot u^{\prime}\right)^{2}+\left(A \cdot S_{L}\right)^{2}\right)^{1 / 2}$. This implies that in the absence of turbulence, for $u^{\prime} \sim 0, S_{T}$ relaxes to $A \cdot S_{L}$. The model parameter $B$ can be determined as follows on a case-by-case basis: 1 ) the mean value of the angle $\theta(h)$ is extracted from the DNS data ( $72 \mathrm{deg}$ for TCF055 and 64.5 deg for TCF150). 2) The value of $S_{T}(h)$ is obtained from Eq. 1 using the mean value of the angle $\theta(h)$. 3) The parameter $B$ is given by $B=\left(\left(S_{T}(h)^{2}-V_{f}^{2}\right) / u^{\prime}(h)^{2}\right)^{1 / 2}$. Figure 5 illustrates a comparison between the model prediction for the wall-distance dependency of the angle $\eta$ and the DNS datasets TCF055 and TCF150 (bottom Xaxis). The normalized turbulent burning velocity $S_{T} / S_{L}$ is also shown on the same plot (top X-axis). $B$ is 2.07 and 3.32 while $y_{b}^{+}$is 14.23 and 17.67 for case TCF055 and TCF150 respectively. The model seems to be able to captures the flame orientation in the bulk region, better so for the TCF055 case than for TCF150. Note that the scatter data shows less spreading, sign of a less turbulent flame, for the case TCF150. The differences in terms of spatial domain size (wall units) and simulation time covered in the two cases imply that the flame propagating in the larger domain, case TCF055, meets a wider pattern of the unsteady near-wall turbulent streaks for a longer time span and it is therefore more turbulent than the flame of case TCF150. Being the present flame-shape model based on a turbulent flame propa-

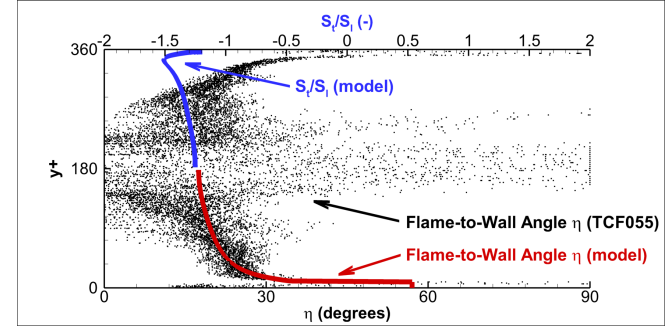

(a)

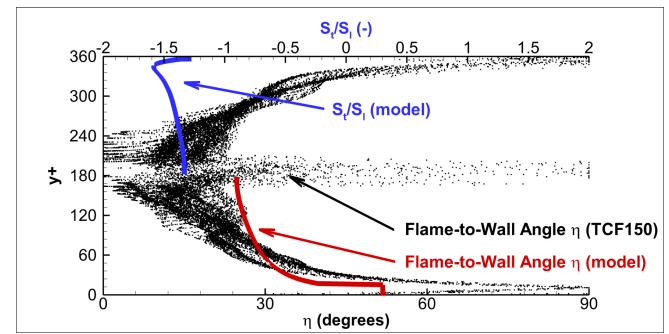

(b)

Figure 5: Comparison between model prediction (solid line, bottom $\mathrm{X}$-axis) and DNS data (scatter plot) for the angle $\eta$ between the flame surface normal and the streamwise direction for case TCF055 5(a) and case TCF150 5(b). The profile of $S_{T}$ as given by the model and normalized by $S_{L}$ is shown in blue (solid line, top X-axis).

gation mechanism (in the bulk) it is not surprising that it performs better for the more turbulent flame of case TCF055. Finally, note also that the model sets the flame angle $\theta$ in the blocked region, for $0<y^{+}<y_{b}^{+}$, to the constant value $\cos \theta=S_{L} / V_{f}=1 / A$ as discussed in Sec. 2. Detailed representation of the flame orientation in the blocked region (DNS data shows a spiky behavior of the angle very close to the wall) is beyond the scope of this simple model that only uses knowledge of the blocked region thickness to adjust $U_{m}(y)$ in the bulk flow above it.

\section{Concluding Remarks and Further Work}

A new model for the mean flame shape during premixed flame flashback in turbulent boundary layers is presented and validated a priori against two DNS datasets. The model is simply constructed as a balance of the mean flow and the propagation of the interface toward the flow, assumes steady state mean flame propagation and takes into account the main features of flashback phenomenology revealed by the DNS. Results indicate that the model is able to capture the mean shape of the turbulent flame brush for both the fuel-lean $(D a<1)$ and the fuel-rich $(D a>1)$ case although the 
prediction in the latter case is somewhat less accurate. Further work is required to validate the model, particularly at higher Reynolds numbers, to assess the scaling of the blocked region thickness for steeper near-wall velocity profiles.

\section{Acknowledgements}

The work at Sandia National Laboratories was supported by the Division of Chemical Sciences, Geosciences, and Biosciences, Office of Basic Energy Sciences of the US Department of Energy and by the US Department of Energy SciDAC Program. SNL is a multiprogramme laboratory operated by Sandia Corporation, a Lockheed Martin Company for the US DOE under Contract DE-AC04-94AL85000. Part of the work is supported by the Combustion Energy Frontier Research Center (CEFRC), an Energy Frontier Research Center funded by the U.S. Department of Energy (DOE), Office of Science, Office of Basic Energy Sciences (BES) under Award No. DESC0001198. Finally, this publication has been produced with support from the BIGCCS Centre, performed under the Norwegian research program Centres for Environment-friendly Energy Research (FME). The authors acknowledge the following partners for their contributions: ConocoPhillips, Gassco, Shell, Statoil, TOTAL, GDF SUEZ and the Research Council of Norway (193816/S60).

\section{References}

[1] K. Döbbeling, J. Hellat and H. Koch, "25 Years of BBC/ABB/Alstom Lean Premix Combustion Technologies", ASME Journal of Engineering for Gas Turbines and Power, 129 (2007) 2-12.

[2] P. Chiesa, G. Lozza, and L. Mazzocchi, "Using Hydrogen as Gas Turbine Fuel", ASME Journal of Engineering for Gas Turbines and Power, 127 (2005) 73-80.

[3] C. Eichler and T. Sattelmayer, "Premixed flame flashback in wall boundary layers studied by long-distance micro-PIV", Experiments in Fluids, 52(2) (2012) 347-360.

[4] A. Gruber, D. Valiev, J.H. Chen and C.K. Law, "Direct numerical simulation of premixed flame boundary layer flashback in turbulent channel flow", Journal of Fluid Mechanics, 709 (2012) $516-542$.

[5] B. Lewis and G. Von Elbe, "Stability and structure of burner flames", Journal of Chemical Physics, 11 (1943) 75-97.

[6] B. Fine, "The Flashback of Laminar and Turbulent Burner Flames at Reduced Pressure", Combustion and Flame, 2 (1958) 253-266.

[7] L. Khitrin, P. Moin, D. Smirnov and V. Shevchuk, "Peculiarities of Laminar- and Turbulent-flame Flashbacks", Tenth Symposium (International) on Combustion, 10 (1965) 1285-1291.

[8] V.N. Kurdyumov, E. Fernández-Tarrazo and A. Liñán, "Flame Flashback and Propagation of Premixed Flames Near a Wall", Proceedings Combustion Institute, 28 (2000) 1883-1889.
[9] V.N. Kurdyumov, E. Fernndez-Tarrazo, J.-M. Truffaut, J. Quinard, A. Wangher and G. Searby, "Experimental and Numerical Study of Premixed Flame Flashback", Proceedings Combustion Institute, 31 (2007) 1275-1282.

[10] V. Bychkov, "Importance of the Darrieus-Landau Instability for Strongly Corrugated Turbulent Flames", Physical Review E, 68(6) (2003) 066304.

[11] S. Chaudhuri, V. Akkerman and C.K. Law, "Spectral Formulation of Turbulent Flame Speed with Consideration of Hydrodynamic Instability”, Physical Review E, 84(2) (2011) 026322.

[12] R. Moser, J. Kim and N. Mansour, "Direct Numerical Simulation of Turbulent Channel Flow up to $R e_{\tau}=590$ ", Physics of Fluids, 11(4) (1999) 943-945.

[13] H. Im and J.H. Chen, "Structure and propagation of triple flames in partially premixed hydrogen-air mixtures", Combustion and Flame, 119 (1999) 436-454.

[14] A. Gruber, P.S. Salimath and J.H. Chen, "Direct numerical simulation of laminar flame-wall interaction for a novel $\mathrm{H} 2$-selective membrane/injector configuration", International Journal of Hydrogen Energy, 39 (2014) 5906-5918.

[15] J. Li, Z. Zhao, A. Kazarov and F.L. Dryer, "An Updated Comprehensive Kinetic Model of Hydrogen Combustion", International Journal of Chemical Kinetics, 36 (2004) 566-575.

[16] T. Poinsot and S.K. Lele, "Boundary Conditions for Direct Simulations of Compressible Viscous Flow", Journal of Computational Physics, 101 (1992) 104-129.

[17] J.C. Sutherland and C.A. Kennedy, "Improved Boundary Conditions for Viscous, Reactive, Compressible Flows", Journal of Computational Physics, 191 (2003) 502-524.

[18] C.S. Yoo and H.G. Im, "Characteristic Boundary Conditions for Simulations of Compressible Reacting Flows with Multidimensional, Viscous and Reaction Effects", Combustion Theory And Modelling, 11(2) (2007) 259-286.

[19] A. Gruber, R. Sankaran, E.R. Hawkes and J.H.Chen, "Turbulent FlameWall Interaction: a Direct Numerical Simulation Study", Journal of Fluid Mechanics, 658 (2010) 5-32.

[20] J.H. Chen, A. Choudhary, B. de Supinski, M. DeVries, E.R. Hawkes, S. Klasky, W.K. Liao, K.L. Ma, J. MellorCrummey, N. Podhorski, R. Sankaran, S. Shende, C.S. Yoo, "Terascale direct numerical simulations of turbulent combustion using S3D”, Computational Science and Discovery, 2 (2009) 131.

[21] C.A. Kennedy and M.H. Carpenter, "Several New Numerical Methods for Compressible Shear-Layer Simulations", Applied Numerical Mathematics, 14 (1994) 397-433.

[22] C.A. Kennedy, M.H. Carpenter and R.M. Lewis, "Low-Storage, Explicit Runge-Kutta Schemes for the Compressible NavierStokes Equations", Applied Numerical Mathematics, 35 (2000) 177-219.

[23] V. Bychkov, "Nonlinear Equation for a Curved Stationary Flame and the Flame Velocity", Physics of Fluids, 10 (1998) 20912098. 